\title{
An Aquatic Insect Imaging System to Automate Insect Classification
}

\author{
M. J. Sarpola, R. K. Paasch, E. N. Mortensen, T. G. Dietterich, \\ D. A. Lytle, A. R. Moldenke, L. G. Shapiro
}

\begin{abstract}
Population counts of aquatic insects are a valuable tool for monitoring the water quality of rivers and streams. However, the handling of samples in the lab for species identification is time consuming and requires specially trained experts. An aquatic insect imaging system was designed as part of a system to automate aquatic insect classification and was tested using several species and size classes of stonefly (Plecoptera). The system uses ethanol to transport specimens via a transparent rectangular tube to a digital camera. A small jet is used to position and reorient the specimens so that sufficient pictures can be taken to classify them with pattern recognition. A mirror system is used to provide a split set of images $90^{\circ}$ apart. The system is evaluated with respect to engineering requirements developed during the research, including image quality, specimen handling, and system usability.
\end{abstract}

Keywords. Automated, Classification, Digital Image, Insects, Water Quality.

A quatic insect population counts are a valuable tool for monitoring environmental quality. The various species and population counts of aquatic insect larva present in a stream provide an effective way to monitor the health of the ecosystem. For example, stonefly (Plecoptera) larvae are very sensitive to changes in water quality. On a scale from 0 to 10 , with 10 being the most tolerant species, stonefly larva rank between 0 and 2 depending on the specific species (Hilsenhoff, 1988). They are usually the first organisms to experience population decline, which make them a good early indicator that water quality is deteriorating. Since stonefly larva live in the stream continuously, they integrate stream health over time and are a better indicator than a single point in time measurement such as chemical analysis (Resh et al., 1996). Several examples of stonefly larva are shown in figure 1.

Collecting and classifying stonefly population counts is time consuming. Several hundred individual insects must be collected and identified for a statistically meaningful population count (Resh et al., 1996). There are about 2000 species of stoneflies worldwide, and a typical stream in North America could have a dozen different species. Accurate classification of species requires an expert who can differentiate the minute differences between some species. People with this

Submitted for review in December 2006 as manuscript number BE 6773; approved for publication by the Biological Engineering Division of ASABE in October 2008.

The authors are Matt J. Sarpola, Engineer, Videx Corporation, Corvallis, Oregon; Robert K. Paasch, School of Mechanical, Industrial, and Manufacturing Engineering, Eric N. Mortensen and Thomas G. Dietterich, School of Electrical Engineering and Computer Science, David A. Lytle, Department of Zoology, and Andrew R. Moldenke, Department of Botany and Plant Pathology, Oregon State University, Corvallis, Oregon; and Linda G. Shapiro, Department of Computer Science and Engineering, University of Washington, Seattle. Corresponding author: Robert K. Paasch, Department of Mechanical Engineering, Oregon State University, Corvallis, OR 97331; phone: 541-737-7019; fax: 541-737-2600; e-mail: paasch@engr.orst.edu.

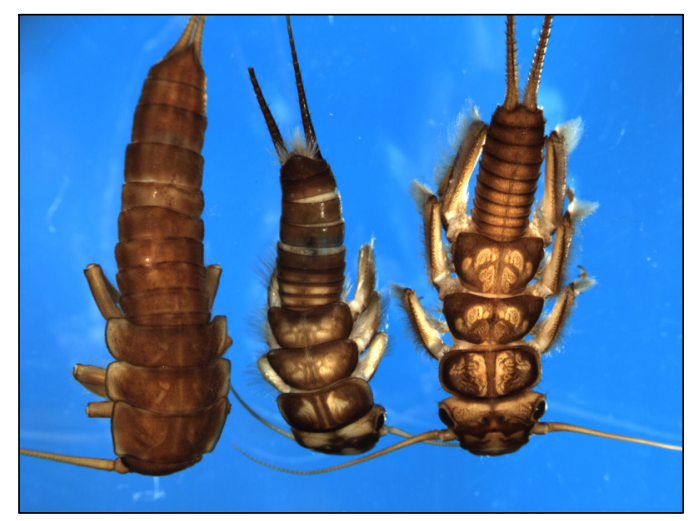

Figure 1. Stonefly larva.

knowledge are in short supply and thus do not have the availability to make insect population counts a viable method of monitoring stream health on a large scale.

To address this challenge, BugID, an automated system for determining stonefly population counts from collected samples has been developed. The objective of the BugID system is to provide expert-level stonefly population counts with minimal operator input and training. The BugID system has two major components: (1) an automated manipulation and imaging system that automatically transports insects to a digital camera and orients them for imaging, and (2) pattern recognition software to classify the species from the digital images.

The objective of this article is to fully describe requirement development for and design and testing of the first major component listed above: an aquatic insect imaging system for automated insect classification. The pattern recognition methods are briefly described in the section below, as the methods used determine many of the requirements for the imaging system. Detailed descriptions of the pattern recognition methods are available in previously published work by the group (Mortensen et al., 2005; Mortensen et al., 2007; 
Zhang et al., 2006; Larios et al., 2007; Deng et al., 2007). The following sections describe relevant literature, the pattern matching approaches and how they impact the imaging system requirements, the development and physical embodiment of the imaging system, and results from testing the imaging system.

\section{Previous Work on Automated Classification of INSECTS}

Automated classification of insects has been attempted with multiple techniques. One technique involves acoustic methods in which insects such as grasshoppers and crickets are classified using signal analysis of the sounds they make (Chesmore and Nellenbach, 2001). Another technique involves using an optical sensor to measure the wingbeat waveforms of mosquitoes (Moore, 1991) and aphids (Moore and Miller, 2002).

The most frequently attempted means of automated insect classification is pattern recognition. Most work on pattern recognition systems involves classifying insects such as bees and wasps by the unique venation in their wings (Yu et al., 1992; Howell et al., 1982; Roth et al., 1999; Weeks et al., 1997; Weeks et al., 1999). However, none of these systems has an automated method for capturing images. Each sample must be prepared individually by hand. This usually involves removing an insect wing and then carefully preparing a slide. A large time investment is required to generate the number of images necessary to allow learning as well as to make meaningful population counts.

\section{Project Overview and Classification APPROACHES}

Development of the BugID system required parallel development of both the imaging system and the classification system. Figure 2 presents a design overview of the entire $\mathrm{Bu}-$ gID system from imaging of specimens to taxonomic identification. The stonefly larvae are prepared and mechanically manipulated for imaging using the hardware, described in detail below. The software control of the mechanical apparatus is integrated with the camera control and imaging software. Images captured by the digital camera are segmented to iden- tify the image regions belonging to the specimen and to separate the specimen from the background. The segmented images are then employed for taxonomic classification in which the goal is to identify each specimen to the species level, although in some cases classification to genus or even just to family is beneficial. The mechanical species manipulation apparatus is designed to simultaneously facilitate two approaches for classification: 2-D pattern matching of the dorsal view, and 3-D specimen reconstruction. To give the reader an idea of the classification techniques used, we give a brief overview of these two approaches.

\section{Two-Dimensional Pattern Matching}

Two-dimensional pattern matching operates directly on one or more images of the specimen. As with many other pattern matching methods, the images are taken from preferred views. In this case, the preferred images are dorsal views that show the patterning, or lack thereof, on the terga (the back plates) of the stonefly larvae, as seen in figure 1 . To facilitate capture of the preferred view, mechanical specimen manipulation provides a means for rotating the specimen until the desired view is obtained. Providing two simultaneous views from different directions increases the likelihood of capturing a good dorsal view. Our 2-D approach (Zhang et al., 2006; Deng et al., 2007) is similar to other recent "bag of parts" methods (Dorkó and Schmid, 2005; Fergus et al., 2005; Opelt et al., 2006) that classify objects from a collection of "interest" region descriptors. The 2-D classification begins by detecting regions of "interest" (areas that are locally unique) and then encoding the local image data around these regions. The methods for detecting and describing the interest regions are robust to changes in viewpoint (up to approximately $30^{\circ}$ to $40^{\circ}$ of out of plane rotation), scale, illumination (i.e., contrast and brightness), and noise. The region descriptors produce a collection of feature vectors, one for each detected interest region, that succinctly and discriminately characterize the local image appearance. In our case, each feature is a 128-dimensional SIFT descriptor (Lowe, 2004; Mortensen et al., 2005) for each interest region.

The images for each species are divided into three sets: one for clustering, one for training the classifier, and one for testing the classifier. All of the feature vectors from all images in the cluster set are used to build "part" clusters, where

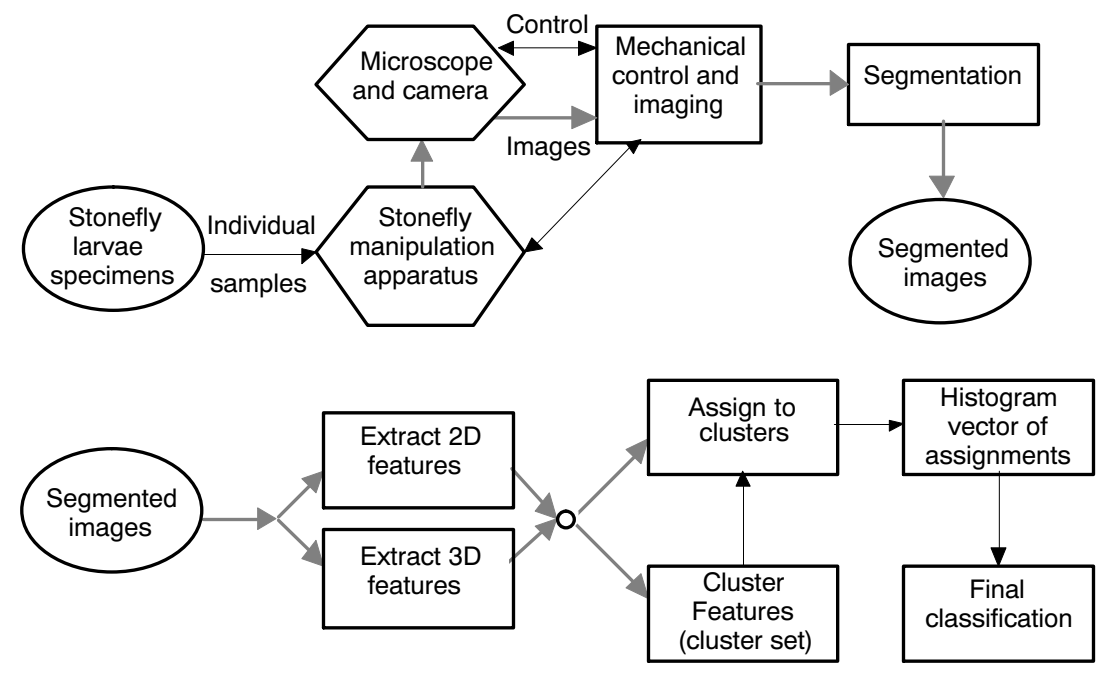

Figure 2. Diagram of stonefly classification system. 
each cluster represents the collection of feature vectors that represent image regions with similar appearance as determined by the SIFT descriptors. For training and classification, each feature vector from an image is assigned to the closest cluster to construct a feature-cluster assignment histogram for each image. The histograms thus constructed, each of which now represents a single vector summarizing the collection of parts detected in an image, are then used for learning a classifier (for training set images) or taxonomic identification (for test set images). Using separate clustering and training data reduces overtraining and improves learning generalization. Details on this approach are available in Larios et al. (2007) and Mortensen et al. (2007).

\section{Three-Dimensional Specimen Reconstruction}

Specimen manipulation is also designed to facilitate three-dimensional reconstruction from a collection of images of a single specimen taken from various viewpoints. The ultimate purpose of 3-D reconstruction is to extract 3-D features similar to the 2-D features described earlier for identification. These 3-D features combine with the 2-D features for specimen classification (fig. 2). The mechanical apparatus was designed particularly to facilitate three reconstruction techniques: (1) multi-view reconstruction of a sparse set of matched feature points, (2) space carving/voxel coloring, and (3) fitting a parameterized 3-D model.

By finding corresponding feature points (i.e., locally unique positions similar to the interest points described previously) across images of the same specimen taken from different viewpoints, multi-view reconstruction computes both the relative camera positions (or equivalently, how the object has moved relative to a fixed camera) and the positions in 3-D space of the matched features. Since reconstruction from a set of sparse points on the specimen does not provide a dense enough 3-D reconstruction, the primary utility of the multiview geometry technique is to provide the relative camera position for each image. Once the camera position is known, more advanced reconstruction techniques, such as space carving and parametric model fitting, can be used.

Space carving (Kutulakos and Seitz, 2000), an extension to voxel coloring (Seitz and Dyer, 1999), computes the "photo hull" of a collection of images taken from known viewpoints. The photo hull is the largest set of voxels (the 3-D equivalent of 2-D image pixels) that are consistent with all the images; as such, the photo hull subsumes the true voxel reconstruction. The photo hull is computed by systematically traversing a 3-D voxel space based on a visibility ordering and determining a consistent coloring for each voxel based on all the images in which the voxel is visible.
A potentially more useful and accurate reconstruction is possible by fitting a parameterized 3D model to the set of images such that, when the rendered 3-D model is virtually projected onto the various camera planes, it produces images that match those of the specimen. The final parameterized model provides a continuous textured surface representation, as opposed to the discrete colored voxels from space carving. However, fitting a parameterized model (Blanz and Vetter, 1999) requires a good initial estimate of the pose and position of the specimen. As such, the voxel positions in the photo hull can be used to initialize the parameterized model (Bardinet et al., 1998).

\section{IMAGING SYSTEM REQUIREMENTS AND SPECIFICATIONS}

The objective of the imaging system was to provide images to the classification system with minimal operator training. In this section, this objective is translated into a definitive set of design requirements, engineering specifications, and target values.

Design requirements for the imaging system are driven by the requirements of the pattern matching approaches and by the needs of the operator. Quantitative engineering specifications and target values for the imaging system were developed from the qualitative design requirements using the Quality Functional Deployment method (Hauser and Clausing, 1988). Initial engineering specifications and target values were ill-defined because of lack of knowledge about both stonefly handling and the pattern matching and reconstruction algorithm capabilities. Refinement of requirements and targets was an iterative process, closely coupled with imaging system and classification algorithm development.

\section{IMAGE QUALITY}

The primary design requirement for the imaging system is image quality. For the purposes of this work, a good image is one that provides a clear, unobstructed view of the stonefly, is sharply focused and well lit, and has good dynamic range without oversaturation. For the stonefly, wide intensity dispersion was desired so that the maximum amount of color and lighting differentiation could be obtained. Standard deviation of the image intensity is used as a measure of dispersion.

Segmentation (digitally removing the background from the stonefly image) requires a uniform blue background, measured by the intensity of the background pixels. In addition, 2-D pattern matching required a dorsal view of the specimen, while 3-D specimen reconstruction required multiple

Table 1. Image quality.

\begin{tabular}{|c|c|c|}
\hline Design Requirements & Engineering Specifications & Targets \\
\hline \multirow[t]{3}{*}{ Clear picture } & Numbers of objects in image other than stonefly (bubbles, scratches, etc.) & $<3$ \\
\hline & Number of objects in image overlaying stonefly & 0 \\
\hline & Number of gill and hair clusters visible (can see individual gills and hairs) & $>2$ \\
\hline Good dorsal view & Rotation of back with respect to image (degrees) & $\pm 15^{\circ}$ \\
\hline \multirow[t]{2}{*}{ Well lighted image } & Image exposure time (ms) & $<250 \mathrm{~ms}$ \\
\hline & Mean stonefly pixel intensity & 100 to 150 \\
\hline Can see entire stonefly & Number of views & 2 at $90^{\circ}$ \\
\hline Easy image segmentation & Median standard deviation of background pixel intensity & $<30$ \\
\hline \multirow[t]{2}{*}{ Stonefly image not saturated } & Median standard deviation of stonefly pixel intensity & $>40$ \\
\hline & Maximum stonefly pixel intensity & $<255$ \\
\hline
\end{tabular}


Table 2. Stonefly handling.

\begin{tabular}{llcc}
\hline Design Requirements & Engineering Specifications & Targets \\
\hline No stonefly damage & Number of appendages lost per stonefly during processing & 0 \\
& Number of body segments damaged per stonefly during processing & 0 & $>3$ \\
\hline Positions stoneflies in minimal time & Time to reorient stonefly (s) & $<10$ & 0 \\
\hline Stoneflies do not jam & Reorientations required for a dorsal view (small/others) & 5 to 20 \\
Works with range of stonefly sizes & Percentage of stoneflies stuck (small/medium/large) & 1 to 5 \\
\hline
\end{tabular}

indexed views of the specimen. From these design requirements, measurable engineering specifications were developed, as shown in table 1. Quantitative target values provide benchmarks against which the success of the imaging system design process can be measured, and were developed to satisfy the image quality levels required by the pattern matching and reconstruction algorithms.

\section{StONEFLY HANDLing}

Stonefly handling is the ability of the system to efficiently and reliably manipulate, orient, and image a wide size range of stoneflies without damaging them. This is a challenging area due to the diversity of stoneflies and their fragile nature.

The specimen collection procedure involves preservation in $70 \%$ ethanol. Removing a specimen from the preservation medium causes it to quickly dry out and curl up unnaturally. The dry specimens are also easily damaged. Imaging a wet specimen in air resulted in oversaturated portions due to glare off the wet surfaces. These results led to the requirement that the specimen remain immersed in a $70 \%$ ethanol solution during imaging.

The two pattern matching approaches have different specimen orientation requirements. The 2-D approach requires good dorsal views of the specimen, whereas the 3-D approach requires multiple indexed views. Specimen reorientation needs to accommodate the full ranges of specimen sizes ( 5 to $20 \mathrm{~mm}$ long for the species in this study) and must be accomplished without specimen damage.

Stoneflies inhabit an environment of flowing water, and have developed physical characteristics such as claws at the ends of their legs that allow them to grip the rocks and debris in the streambed. These characteristics make stoneflies prone to snagging on any discontinuity or roughness within the imaging system.

From these design requirements, measurable engineering specifications for specimen handling were developed, as shown in table 2. Target values reflect the need to minimize specimen damage and handling time, and to accommodate the expected range of specimen sizes.

\section{IMAGING SySTEM DEVELOPMENT}

Development of an imaging system meeting the design requirements required multiple iterations. This section provides a physical description of the final aquatic insect imaging system. The imaging system, shown in figures 3 and 4, consists of the following major components:

- MicroPublisher 5.0 RTV 5 megapixel color digital camera (QImaging Corp., Surrey, BC, Canada).

- Leica MZ9.5 high-performance stereomicroscope with 0.32 objective (Leica Microsystems GmbH, Wetzlar, Germany).
- Two Volpi V-Lux 1000 cold light sources with gooseneck light guides and diffusers (Volpi AG, Schlieren, Switzerland).

- Aquatic insect transport and orientation device with pumps (Super X DC pumps, Sonic-Tronics, Inc., Elkins Park, Pa) and a microcontroller (ATmega8535, Atmel Corp., San Jose, Cal.).

- Mirror system providing split images of the specimens.

- Infrared sensor (Tekbot IR wheel encoder board, School of Electrical Engineering and Computer Science, Oregon State University, Corvallis, Ore.).

- Calibration object (glass fixed-frequency grid distortion target, $25 \times 25$ dot grid on $0.25 \mathrm{~mm}$ centers, Edmunds Optics, Barrington, N.J.).

- Desktop computer to control the camera and control board (Optiplex 745, Dell Computer Corp., Round Rock, Tex.).

The aquatic insect transport and orientation device and mirror system are mounted below the microscope and camera for image capture, as shown in figure 4 . The digital camera is attached to a Leica MZ9.5 high-performance stereomicroscope at $0.63 \times$ magnification, providing a resolution of approximately 75 pixels $\mathrm{mm}^{-1}$. A 0.32 objective is used on the microscope to increase field of view, depth of field, and working distance. All images are lighted using gooseneck light guides powered by Volpi V-Lux 1000 cold light sources. Diffusers on the ends of the light guides eliminate hard shadows and reduce specular reflections that typically cause image saturation. Lighting and exposure time are set at fixed values for all specimens to avoid any influence on the classification.

The aquatic insect transport and orientation device has several components. The main mechanical system is made of abrasion-resistant polycarbonate and high-density polyethylene (both materials are resistant to alcohol). It consists

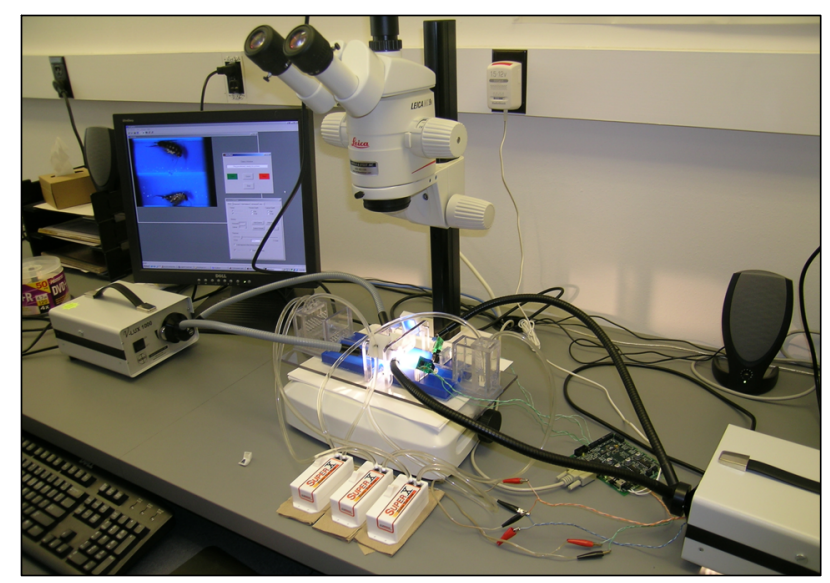

Figure 3. Aquatic insect imaging system. 


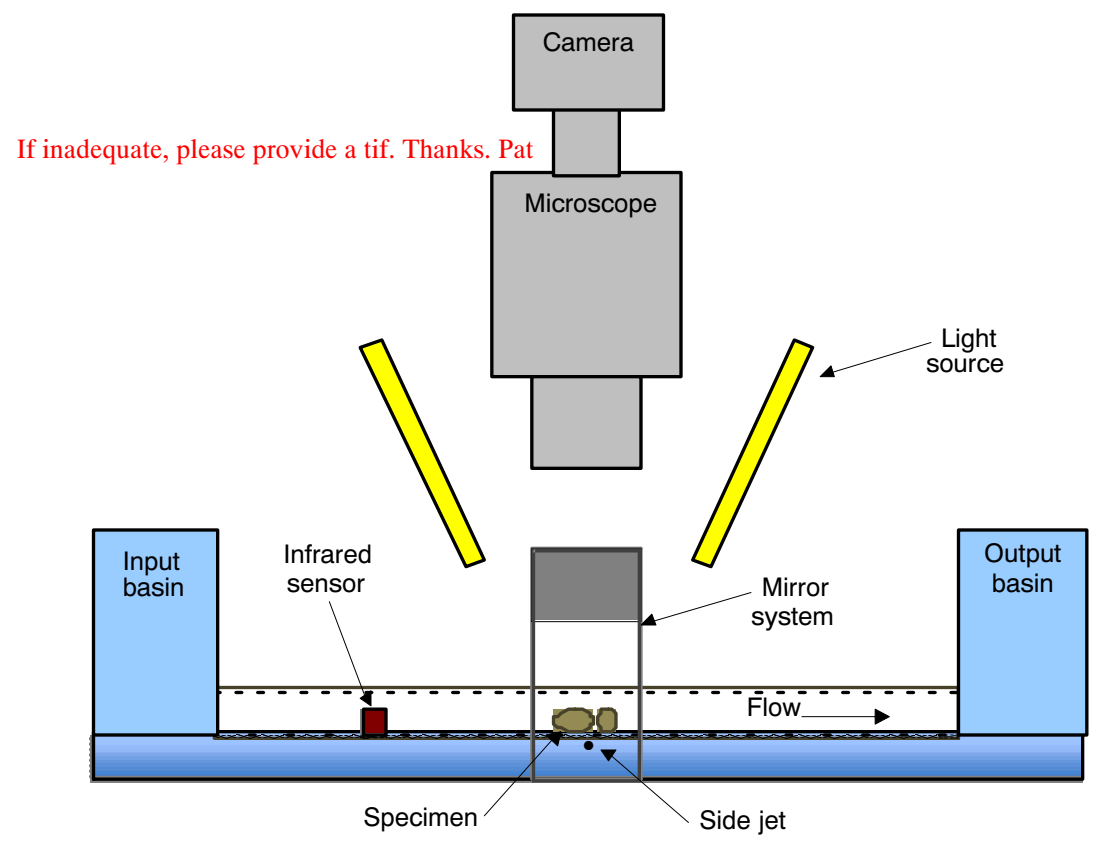

Figure 4. Aquatic insect imaging system.

of two basins connected by a square tube, $12 \times 12 \mathrm{~mm}$ inside dimension. The tube is rotated $45^{\circ}$ from horizontal, with transparent polycarbonate on the two top sides and blue polyethylene on the two bottom sides. Filling the two basins and tube with $70 \%$ ethanol, and pumping the fluid from one basin to the other, creates a longitudinal flow inside the tube to transport the stoneflies to the imaging area under the microscope. A benefit of the $70 \%$ ethanol solution is its low surface tension, which reduces bubble formation. Bubbles collecting in the imaging area make classification difficult by obstructing the stonefly.

A small side jet at the center of the tube is used to stop and orient the stoneflies as they pass through the tube. The jet is $0.5 \mathrm{~mm}$ (0.020 in.) in diameter. The side jet creates a vortex that causes the stoneflies to spin in place within the imaging area. The longitudinal flow is stopped once the stoneflies are caught in the jet, and then the jet is turned off and the stone- flies settle to the bottom of the tube where the image is captured. Pulsing the jet reorients stoneflies to provide new views for both 2-D identification and 3-D reconstruction.

The mirror system can be seen in figure 5. This mirror system fits over the image area at the center of the tube, below the microscope and camera. The mirrors provide a split image, with two views of the insect approximately $90^{\circ}$ apart. Providing two simultaneous views increases the likelihood of good dorsal views for 2-D classification, and it affords greater robustness for 3-D reconstruction since the camera viewpoints of the two views are fixed. The exact relative (virtual) camera position for each split view is determined by imaging a calibration object with markings that have known geometry. The calibration object (also shown in fig. 5) fits into the tube and is manipulated in a manner similar to the stonefly specimens.
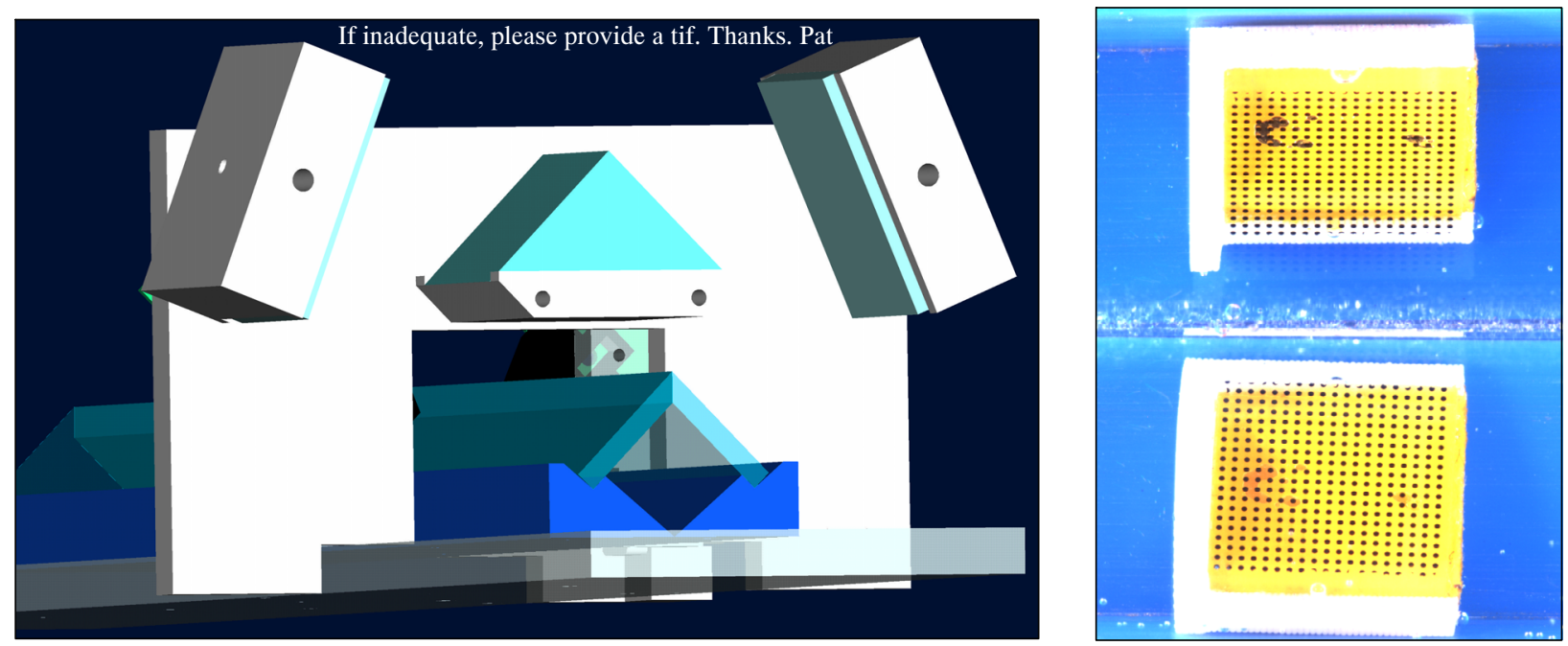

Figure 5. Mirror configuration and split image of calibration object. (Don't know why there was a color change upon importing. Pat) 
The transport and orientation device is automated using an Atmel ATmega8535 microcontroller. Two infrared sensors were used to detect the presence of a stonefly in the tube. Once a stonefly is detected, the microcontroller automatically controls the side jet and longitudinal flow to stop the stonefly in the imaging area. Then a graphical user interface on the desktop computer is used to communicate with the system via a serial connection. Imaging and stonefly reorientation are performed through the graphical user interface.

For each image capture session, the system is first calibrated by dropping the calibration object into the entry basin, transporting it to the imaging area, and capturing an image. A background-only image is also captured to aid segmentation. In operation, an individual specimen is dropped into the entry basin, and the system is notified through the graphical user interface. The system transports the specimen down the tube. When the infrared sensors detect the insect, the system turns off the longitudinal flow and, after a $30 \mathrm{~ms}$ delay, turns on the side jet to capture the specimen in the imaging area. The side jet is turned off, the insect settles to the bottom of the tube, and a split image captured. The side jet is then pulsed to reorient the insect about its roll axis, and another image is captured. After imaging, the insect is transported to the exit basin and removed.

\section{Test Methodology}

\section{IMAGING SYSTEM}

The performance of the aquatic insect imaging system was tested in order to determine if the system met the engineering specification targets. Testing included the imaging of 300 individual stonefly specimens. Three common species were used for testing: Calineuria californica, Doroneuria baumanni, and Hesperoperla pacifica; small, medium, and large specimen sizes were tested for each species. The digital images of the specimens were stored on the desktop computer for later processing.

A MATLAB program was written to quantify the image quality. This program first segmented the stonefly from the background using color information. Then, for each pixel, the intensities of the three color channels were averaged to give a single intensity value for each pixel. The program created histograms of the background and stonefly pixel intensity values, which ranged from 0 to 255 for these 8-bit images. The procedure allowed for a qualitative evaluation of how well the program performed at segmenting the stonefly, and it allowed a quantitative evaluation of the uniformity of the background and dynamic range of the stonefly image. Image quality statistics are based on segmentation and analysis of 55 images.

For each imaged specimen, observations were recorded regarding the handling of the specimen by the system. These observations included whether the specimen was damaged by the system, the time to reorient the specimen, the number of reorientations needed to obtain a dorsal view, whether the specimen became stuck in the system, and the length and width of each specimen.

\section{Stonefly Classification}

Once the imaging system was optimized, we conducted experiments with the two classification approaches. Preliminary classification results from 3-D reconstruction were not promising, and this approach was abandoned in favor of a focused effort on the 2-D approach. For 2-D pattern matching, three experiments were conducted on stonefly classification from images produced by the imaging system, as reported by Mortensen et al. (2007). In each experiment, we trained the classification system to discriminate between two species. The three experiments presented progressively increasing levels of difficulty:

1. The somewhat easy task of discriminating between the very distinctive Calineuria californica and Yoraperla sp.

2. The moderately difficult task of discriminating between Hesperoperla pacifica and Doroneuria baumanni.

3. The hard task of discriminating between the very similar Calineuria californica and Doroneuria baumanni.

These experiments were performed as follows: the image dataset was randomly divided into three completely disjoint sets of equal size. To avoid any kind of influence in the test results, different images of the same insect instance were placed in the same set. The first set was used as the "clustering set" to create the clusters for each object class, the second set was used to train the 15 decision trees that comprise the final classifier, and the third was then used to measure the classification accuracy of the classifier.

\section{Test Results}

\section{IMAGE QUALITY}

Example images before and after segmentation are shown in figure 6. Histograms of pixel intensity for this image are shown in figure 7 . Target values and test results for each engineering specification for image quality are shown in table 3 . These can be compared directly to indicate specification satisfaction.

All but one of the 55 images segmented had background dispersions less than 40, with a median dispersion of 27.5. This shows that the backgrounds are sufficiently uniform. Segmentation results also confirm that the background is sufficiently uniform. The segmentation program was able to extract the majority of the stoneflies from the image. Darker parts of the stonefly, such as the eyes and other areas on the head, proved to be the most difficult to extract.

Due to optimization of exposure time, none of the images from the system showed any saturation. It is acceptable to reduce the image exposure time so that no saturation occurs, even though this also reduces the dynamic range of the stonefly. Information is completely lost when saturation occurs, so this situation must be avoided. The dynamic range of the stonefly can be increased through image processing procedures such as histogram equalization if necessary.

\section{STONEFLY HANDLING}

Targets and evaluation results for stonefly handling are shown in table 4 . The imaging system met all requirements for damage; fluidic handling of the stoneflies produced essentially no damage to the specimens. The side jet repositioned the specimens for imaging very rapidly, but because of the tendency of the specimens to land on their back, sometimes many reorientations were required to achieve a good dorsal view of a specimen. This was particularly true of small 

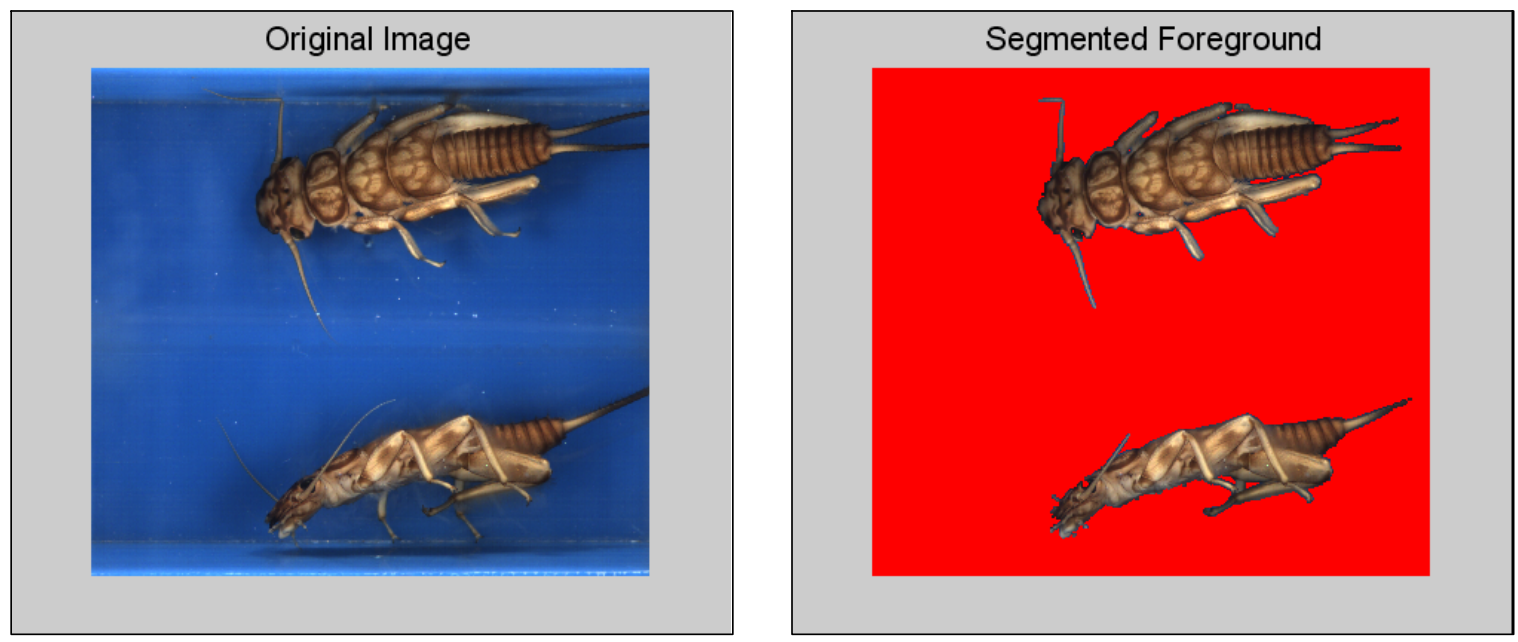

Figure 6. Example images.
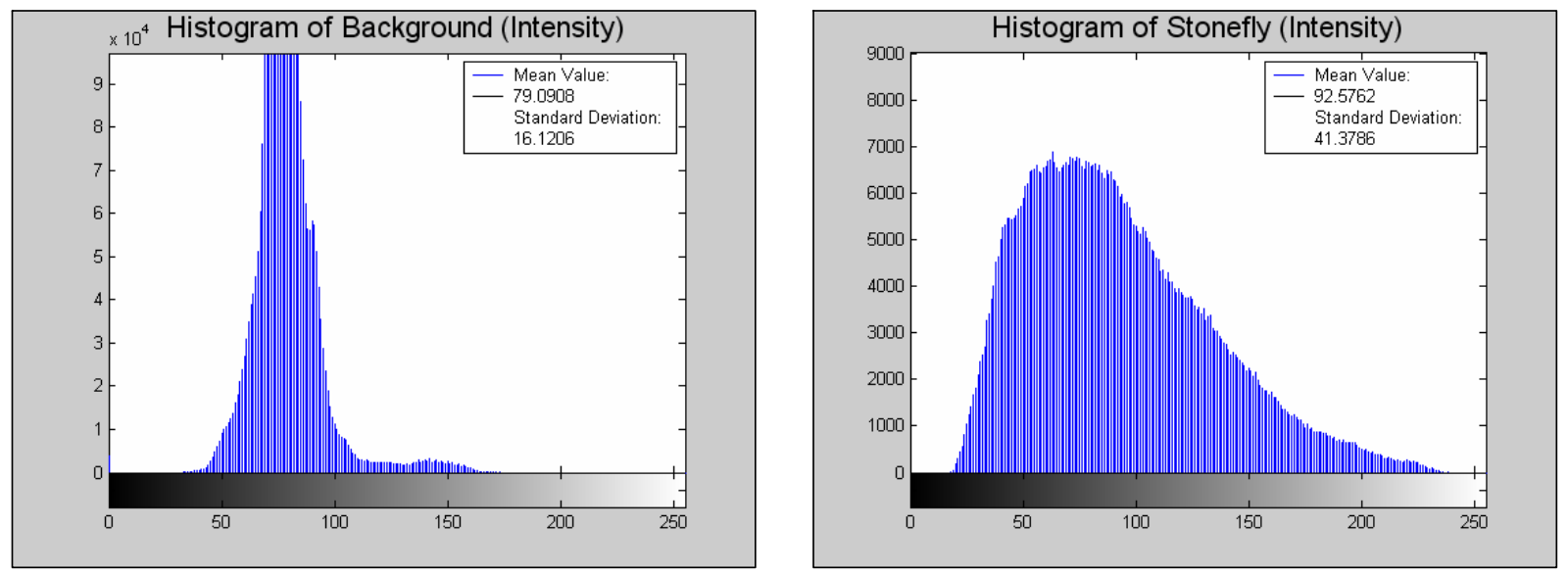

Figure 7. Image analysis results.

Table 3. Image quality.

\begin{tabular}{llcc}
\hline Design Requirements & Engineering Specifications & Targets & Results \\
\hline Clear picture & Numbers of objects in image other than stonefly (bubbles, scratches, etc.) & $<3$ & 0 \\
& Number of objects in image overlaying stonefly & 0 & 0 \\
& Number of gill and hair clusters visible (can see individual gills and hairs) & $>2$ & 7 \\
\hline Good dorsal view & Rotation of back with respect to image (degrees) & $\pm 15^{\circ}$ & $<5^{\circ}$ \\
\hline Well lighted image & Image exposure time (ms) & $<250 \mathrm{~ms}$ & $245 \mathrm{~ms}$ \\
& Mean stonefly pixel intensity & 100 to 150 & 124 \\
\hline Can see entire stonefly & Number of views & 2 at $90^{\circ}$ & 2 at $90^{\circ}$ \\
\hline Easy image segmentation & Median standard deviation of background pixel intensity & $<30$ & 27.5 \\
\hline Stonefly image not saturated & Median standard deviation of stonefly pixel intensity & $>40$ & 43.4 \\
& Maximum stonefly pixel intensity & $<255$ & 254 \\
\hline
\end{tabular}

Table 4. Stonefly handling.

\begin{tabular}{llcc}
\hline Design Requirements & Engineering Specifications & Targets & Results \\
\hline No stonefly damage & Number of appendages lost per stonefly during processing & 0 & 0 \\
& Number of body segments damaged per stonefly during processing & 0 & 0 \\
\hline Positions stoneflies in minimal time & Time to reorient stonefly (s) & $>3$ & $<10$ \\
& Reorientations required for a dorsal view (small/others) & $>10 / 5$ 远10 \\
\hline Stoneflies do not jam & Percentage of stoneflies stuck (small/medium/large) & 0 & $0 / 15 / 25$ \\
\hline Works with range of stonefly sizes & Range of stonefly lengths that system can effectively process (mm) & 5 to 20 & 8 to 12 \\
& Range of stonefly widths that system can effectively process (mm) & 1 to 5 & 2 to 4 \\
\hline
\end{tabular}


Table 5. Classification results using decision trees (Mortensen et al., 2007).

\begin{tabular}{lc}
\hline Experiment & Classification Accuracy (\%) \\
\hline 1. Calineuria vs. Yoraperla & $94.4 \pm 2.6$ \\
2. Hesperoperla vs. Doroneuria & $90.4 \pm 3.3$ \\
3. Calineuria vs. Doroneuria & $73.3 \pm 6.9$ \\
\hline
\end{tabular}

specimens. The system thus did not meet the target for number of reorientations.

Although care was taken in design and construction to mount the infrared detector mirrors flush to the tube surface with no gaps, some of the larger stoneflies still snagged on the mirrors. There were also some specimens that snagged on the $0.5 \mathrm{~mm}$ (0.020 in.) diameter side jet hole. This limited the effective upper range for size of specimens that the system would process efficiently.

\section{Stonefly Classification}

Table 5 presents the resulting accuracy rates for our three classification experiments (along with $95 \%$ confidence intervals). For experiment 1 (Calineuria vs. Yoraperla), the method achieved 94\% accuracy; for experiment 2 (Hesperoperla vs. Doroneuria), the accuracy was $90 \%$, and for the very difficult experiment 3 (Calineuria vs. Doroneuria), the method attained $73 \%$ correct classification.

\section{SUMMARY}

This article has described a hardware and software system for automated stonefly classification. The goal of the system was to provide rapid, automated classification of stonefly larvae to facilitate the ecological monitoring of freshwater streams. Two software approaches for classification were described: 2-D pattern matching of the dorsal view, and 3-D specimen reconstruction. The detailed development and testing of the image capture system was also described.

The imaging system successfully produced images of consistently high quality, meeting all engineering specifications for image quality. With some exceptions, specimen handling and system usability were satisfactory. Handling of stonefly larvae presents significant challenges due to the requirement that they remain immersed in liquid, and due to their physical structure and diversity. Fluidic handling of the specimens prevented any damage, but large specimens showed an occasional tendency to jam in the transport tube, and small specimens required more reorientations than desired. System automation allowed minimal operator input and high sample throughput.

Current efforts are directed toward integration of the hardware-software system and continued refinement of the pattern matching algorithms. Currently, the process of image capture continues until the operator deems sufficient images of that particular specimen have been captured. Once the imaging and classification systems are integrated, imaging would continue only until the specimen is identified.

The objective of the BugID system is to provide expertlevel stonefly population counts with minimal operator input and training. The BugID system shows promise in classifying stoneflies to the species level, but there is significant room for improvement. We continue to explore classification methods to this end.

\section{ACKNOWLEDGEMENTS}

The authors gratefully acknowledge the support of the U.S. National Science Foundation under Grant No. IIS-0326052. The views expressed are those of the authors and do not represent the views of the U.S. Government or the National Science Foundation.

\section{REFERENCES}

Bardinet, E., L. D. Cohen, and N. Ayache. 1998. A parametric deformable model to fit unstructured 3D data. Computer Vision and Image Understanding 71(1): 39-54.

Blanz, V., and T. Vetter. 1999. A morphable model for the synthesis of 3D faces. In Proc. Computer Graphics (SIGGRAPH), 187-194. Association for Computing Machinery, Special Interest Group on Graphics and Interactive Techniques.

Chesmore, E. D., and C. Nellenbach. 2001. Acoustic methods for the automated detection and identification of insects. Acta Hort. 562: 223-231.

Deng, H., W. Zhang, E. N. Mortensen, T. G. Dietterich, and L. G. Shapiro. 2007. Principal curvature-based region detector for object recognition. In Proc. Conf. on Computer Vision and Pattern Matching (CVPR '07). Piscataway, N.J.: IEEE.

Dorkó, G., and C. Schmid. 2005. Object class recognition using discriminative local features. Technical Report RR-5497. Montbonnot, France: INRIA-Grenoble, Rhone-Alpes Research Center.

Fergus, R., P. Perona, and A. Zisserman. 2005. A sparse object category model for efficient learning and exhaustive recognition. In Proc. Conf. on Computer Vision and Pattern Recognition (CVPR '05), I: 380-387. Piscataway, N.J.: IEEE.

Hauser, J., and D. Clausing. 1988. The house of quality. Harvard Business Review (May-June): 63-70.

Hilsenhoff, W. L. 1988. Rapid field assessment of organic pollution with a family-level biotic index. J. North American Benth. Soc. 7(1): 65-68.

Howell, V. D., K. Hoelmer, and P. A. Norman. 1982. Computer-assisted measurement and identification of honey bees. Ann. Entomol. Soc. of America 75(6): 591-594.

Kutulakos, K. N., and S. M Seitz. 2000. A theory of shape by space carving. Intl. J. Computer Vision 38(3): 199-218.

Larios, N., H. Deng, W. Zhang, M. Sarpola, J. Yuen, R. K. Paasch, A. Moldenke, D. A. Lytle, S. Ruiz Correa, E. N. Mortensen, L. G. Shapiro, and T. G. Dietterich. 2007. Automated insect identification through concatenated histograms of local appearance features. In IEEE Workshop on Applications of Computer Vision (WACV 2007), 23-32. Piscataway, N.J.: IEEE.

Lowe, D. G. 2004. Distinctive image features from scale-invariant keypoints. Intl. J. Computer Vision 60(2): 91-110.

Moore, A. 1991. Artificial neural network trained to identify mosquitoes in flight. J. Insect Behavior 4(3): 391-396.

Moore, A., and R. Miller. 2002. Automated identification of optically sensed aphid wingbeat waveforms. Ann. Entomol. Soc. of America 95(1): 1-8.

Mortensen, E. N., H. Deng, and L. Shapiro. 2005. A SIFT descriptor with global context. In Proc. Conf. on Computer Vision and Pattern Recognition (CVPR '05), I: 184-190. Piscataway, N.J.: IEEE.

Mortensen, E. N., E. L. Delgado, H. Deng, D. Lytle, A. Moldenke, R. Paasch, L. Shapiro, P. Wu, W. Zhang, and T. G. Dietterich. 2007. Pattern recognition for ecological science and environmental monitoring: An initial report. In Automated Taxon Identification in Systematics, 189-206. N. MacLeod and M. O’Neill, eds. Boca Raton, Fla.: CRC Press.

Opelt, A., A. Pinz, and A. Zisserman. 2006. Incremental learning of object detectors using a visual shape alphabet. In Proc. Conf. on Computer Vision and Pattern Recognition (CVPR '06), I: 3-10. Piscataway, N.J.: IEEE. 
Resh, V. H., M. J. Myers, and M. J. Hannaford. 1996. Macroinvertebrates as biotic indicators of environmental quality. In Methods in Stream Ecology, 647-667. F. R. Hauer and G. A. Lamberti, eds. San Diego, Cal.: Academic Press.

Roth, V., V. Steinhage, S. Schroder, A. B. Cremers, and D. Wittmann. 1999. Pattern recognition combining de-noising and linear discriminate analysis within a real-world application. Lecture Notes in Computer Sci. 1689: 251-258.

Seitz, S. M., and C. R. Dyer. 1999. Photorealistic scene reconstruction by voxel coloring. Intl. J. Computer Vision 35(2): 151-173.
Weeks, P. J. D., I. D. Gauld, K. J. Gaston, and M. A. O’Neill. 1997. Automating the identification of insects: A new solution to an old problem. Bulletin Entomol. Res. 87(2): 203-211.

Weeks, P. J. D., M. A. O'Neill, K. J. Gaston, and I. D. Gauld. 1999. Automating insect identification: Exploring the limitations of a prototype system. J. Applied Entomol. 123(1): 1-8.

Yu, D. S., E. G. Kokko, J. R. Barron, G. B. Schaalje, and B. E. Gowen Dicky. 1992. Identification of ichneumonid wasps using image analysis of wings. Systematic Entomol. 17(4): 389-395.

Zhang, W., H. Deng, T. G. Dietterich, and E. N. Mortensen. 2006. A hierarchical object recognition system based on multi-scale principal curvature regions. In Proc. Intl. Conf. on Pattern Recognition (ICPR), I: 778-782. Piscataway, N.J.: IEEE. 
